Results 55 participants reported with a median time of 63 days (95\%CI: 35-133) a severe health problem. Women had significantly $(\mathrm{p}=0.02)$ shorter median time to first health problem (35 days; 95\%CI: 7-105) compared to men (98 days; 95\%CI: 49-294) and nearly twice as high risk to sustain a severe health problem (HR: 1.88; 95\%CI: 1.1-3.24). Age or impairment comparisons showed no significant differences. Injuries at the shoulder resulted in the highest burden with 6.5 time loss days per athlete per year followed by the hand (2.9) and trunk (2.6). Respiratory infections showed the highest burden with 5.2 time loss days per athlete per year followed by genitourinary illnesses (1.9) and infectious diseases (1.8).

Conclusion Sex but not age or impairment type showed significant differences on time to health problem and enhanced risks. Upper body injuries and respiratory infections generated high burden.

\section{INJURY AND ILLNESS IN ELITE ATHLETICS: A PROSPECTIVE COHORT STUDY OVER THREE SEASONS}

${ }^{1}$ Shane Kelly, ${ }^{2}$ Noel Pollock, ${ }^{3}$ George Polglass, ${ }^{4}$ Ben Clarsen. ${ }^{1}$ Ballet Healthcare, Royal Opera House, London, UK; ${ }^{2}$ Institute of Sport Exercise and Health, London, UK; ${ }^{3}$ British Athletics Medical Department, Loughborough, UK; ${ }^{4}$ Oslo Sports Trauma Research Center, Department of Sports Medicine, Norwegian School of Sports Sciences, Oslo, Norway

\subsection{6/bjsports-2021-IOC.410}

Background Athletics is one of the most popular sports in the world and is the centrepiece of the Summer Olympic Games. Participation in athletics training and competition involves a risk of illness and injury.

Objective This paper reports and summarises injury and illness in British Olympic track and field athletes over three full training and competition seasons.

Study Design Descriptive epidemiological study

Setting Elite athletics training centres

Participants Elite track and field athletes from the British national programme

Main outcome measures Exposure, incidence, severity, burden, mechanism- acute vs overdue

Methods A total of 111 athletes on the British national programme were followed prospectively for three consecutive seasons between 2015-2018. Team medical personnel recorded all injuries and illnesses during this time, following current consensus-based methods.

Results The average age of the athletes was 24 years for both males and females (24 years, SD: 4). Total exposure for the three seasons was 79205 athlete days (217 athlete years). Overuse injuries $(56.4 \%)$ were more frequent than acute injuries $(43.6 \%)$. The thigh was the most common injury location (0.6 per athlete year), followed by the lower leg (0.4 per athlete year) and foot ( 0.3 per athlete year). Muscle and tendon were the most commonly injured tissues, while strains and tears were the most common pathology type. Respiratory illness was the most common illness type ( 0.3 per athlete year). Hamstring muscle strain was the most common diagnosis causing time loss, followed by Achilles tendinopathy and soleus muscle strain.

Conclusion Our findings indicate that future injury prevention efforts within elite athletics need to focus specifically on hamstring strains, Achilles tendinopathy, and soleus strains. Improved knowledge of the aetiology and risk profile of these problems in elite athletes is needed. REVIEW WITH QUALITY ASSESSMENT

${ }^{1}$ Sietske Luijten, ${ }^{2}$ Leonie te Loo, ${ }^{1}$ Joske Nauta, ${ }^{3}$ Thomas Janssen, ${ }^{4,5}$ Jasmijn Holla, ${ }^{6}$ René Otten, ${ }^{1,7}$ Ingrid Vriend, ${ }^{1,7}$ Evert Verhagen. ${ }^{1}$ Departement of public and occupational health, Amsterdam UMC, Amsterdam, Netherlands; ${ }^{2}$ Sport Studies, Inholland University of Applied Sciences, Haarlem, Netherlands; ${ }^{3}$ Faculty of behavioural and movement sciences, Vrije Universiteit, Amsterdam, Netherlands; ${ }^{4}$ Faculty of Health, Sports and Social Work, Inholland University of Applied Sciences, Haarlem, Netherlands; ${ }^{5}$ Amsterdam Rehabilitation Research Center, Reade, Amsterdam, Netherlands; ${ }^{6}$ Medical Library, Vrije Universiteit, Amsterdam, Netherlands; ${ }^{7}$ Amsterdam Public Health research institute, Amsterdam, Netherlands

\subsection{6/bjsports-2021-IOC.411}

Background Participation is sport is associated with a risk of sports injuries and illnesses. For athletes with an impairment, sports related health issues pose further burden upon an already restricted lifestyle. This underlines the importance of injury prevention in adapted sports.

Objective To provide an overview of the current evidence regarding injuries and their prevention in adapted sports.

Design A systematic review with quality assessment.

Setting Peer-reviewed literature on sports injuries in adapted sports.

Participants Individuals with a physical impairment that affects motor function, and who are active in sports or physical activity. Assessment of Risk Factors This study was conducted in accordance with the 'Preferred Reporting Items for Systematic Reviews and Meta-Analyses' (PRISMA) guidelines.

Main Outcome Measurements Literature and evidence was categorised by the sequence of prevention; i.e. (1) problem magnitude; (2) aetiology of injury; (3) development of preventive measures; and (4) evaluation of effectiveness.

Results 52 studies were included. A total of 5 studies reported on the first step of the sequence of prevention (problem magnitude) only. 28 studies reported information on both the first and second step, 15 studies on only the second step and only 4 studies on the third and fourth step of the sequence. Most studies included participants of an elite level (82.7\%). There is a wide range of injury and illness incidence between various sports (2.2 - 90.9 per 1000 athlete days) and impairment categories (0.6 - 50.0 per 1000 athlete days).

Conclusions Current evidence regarding injuries in adapted sports is mostly limited to elite level athletes. The evidence regarding the development of preventive measures and their effectiveness is limited in this target group. More knowledge is needed of the aetiology and risk factors of various adapted sports, physical impairments and level of performance to develop future prevention strategies for this population.

\section{INJURY RATES, TYPES AND MECHANISMS IN SLEDGE HOCKEY: IMPLICATIONS FOR GRASSROOTS THROUGH ELITE PARTICIPATION}

${ }^{1}$ Alexandra J Sobry, ${ }^{1}$ Ash T Kolstad, 'Leticia Janzen, ${ }^{1,2,3,4}$ Amanda M Black, 1,2,3,4,5,6,7 Carolyn A Emery. 'Sport Injury Prevention Research Centre, Faculty of Kinesiology, University of Calgary, Calgary, Canada; ${ }^{2}$ Alberta Children's Hospital Research Institute, University of Calgary, Calgary, Canada; ${ }^{3} \mathrm{O}^{\prime}$ Brien Institute for Public Health, University of Calgary, Calgary, Canada; ${ }^{4}$ Hotchkiss Brain Institute, University of Calgary, Calgary, Canada; ${ }^{5}$ McCaig Institute for Bone and Joint Health, University of Calgary, Calgary, Canada; ${ }^{6}$ Community Health Sciences, Cumming School of Medicine, University of Calgary, Calgary, Canada; 'Department of Pediatrics, Cumming School of Medicine, University of Calgary, Calgary, Canada

10.1136/bjsports-2021-IOC.412 
Background Injuries in parasport are not well explored and may lead to detrimental effects in players with pre-existing disabilities. Previous parasport injury studies have focused on elite sledge hockey during winter Paralympics. Reported injury rates in sledge hockey are higher than other winter parasports.

Objective To examine concussion and injury rates, locations, types, mechanisms, and risk factors in sledge hockey.

Design Cross-sectional survey.

Setting Sledge hockey players across all levels of play.

Participants Sledge hockey players (ages $\geq 14$ years) recruited worldwide following the 2019-2020 season through email, social media and word of mouth communication. Ninety-two players initiated the survey and 77 (83.7\%) answered questions related to sledge hockey injuries.

Assessment of Risk Factors Risk factors considered participant demographics (e.g., age, sex, disability, level of play).

Main Outcome Measurements One-year injury rates (IR) and incidence rate ratios (IRR) were estimated based on univariate Poisson regression analyses. Injury proportions by type, location, and mechanism were described.

Results There were 47 injuries reported including 16 concussions in $9 / 77(11.7 \%)$ players and 31 non-concussion injuries in $20 / 77(26.0 \%)$ players. The overall IR was 13.2 injuries/ 1000 athlete-exposures (95\%CI;9.6-17.6). The game IR (28.4 injuries/1000 game-exposures, 95\%CI;18.6-41.7) was significantly higher than for practices (4.4 injuries/1000 practiceexposures, 95\%CI;2.2-7.9) $(\mathrm{IRR}=6.5$, 95\%CI;3.1-14.5). Significant injury: (1) locations were the head (34.0\%), wrist/ hand $(14.8 \%)$ and shoulder $(10.6 \%)$; (2) types were concussion (36.2\%) and bone fracture (8.5\%); and (3) mechanism was body checking $(42.1 \%$ of injuries caused by contact with another player). Age, sex, disability type, and level of play were not found to be injury risk factors.

Conclusions Concussions and upper extremity injuries were the most common sledge hockey injuries reported, with body checking being the most common mechanism. This research will inform the development of sledge hockey injury prevention strategies.

\section{WHO? WHAT? WHERE? WHY? DESCRIBING THE PATTERNS OF INJURY IN HIGH SCHOOL MALE COLLISION SPORTS}

\begin{abstract}
1,2Stephen West, ${ }^{1}$ Eric Gibson, ${ }^{1}$ Mark Patrick Pankow, 1,2,3,4Amanda Black, 1,2,3,4,5,6,7 Carolyn Emery. 'Sport Injury Prevention Research Centre, Faculty of Kinesiology, University of Calgary, Canada, Calgary, Canada; ${ }^{2} \mathrm{O}$ Brien Institute of Public Health, University of Calgary, Calgary, Canada; ${ }^{3}$ Alberta Children's Hospital Research Institute, University of Calgary, Canada, Calgary, Canada; ${ }^{4}$ Hotchkiss Brain Institute, University of Calgary, Canada, Calgary, Canada; ${ }^{5}$ McCaig Institute for Bone and Joint Health, University of Calgary, Canada, Calgary, Canada; ${ }^{6}$ Community Health Sciences, Cumming School of Medicine, University of Calgary, Canada, Calgary, Canada; 'Department of Paediatrics, Cumming School of Medicine, University of Calgary, Canada, Calgary, Canada
\end{abstract}

\subsection{6/bjsports-2021-IOC.413}

Background Collision sports (Canadian football, ice hockey, lacrosse, rugby) are popular among Canadian male youth, however it is recognised that collision sports are associated with a high risk of injury.

Objective To describe the patterns of collision sport-related injury in Canadian male high school athletes.

Design Secondary analysis of a cross-sectional survey.

Setting High schools (Alberta, Canada)
Participants 360 male students (of 2029 respondents), who play at least one of Football, Hockey, Lacrosse or Rugby.

Assessment of Risk Factors An anonymous online survey included questions regarding the mechanism, site, type, and nature of collision sport injuries.

Main Outcome Measurements Sport-related injury self-reported in the past year.

Results Of the 2029 survey respondents, 958 (47.2\%) were male of which $360(37.6 \%)$ reported playing at least one collision sport. Of all serious injuries reported by males, collision sports accounted for 33\% [hockey: 63(17\%), football: 41(11\%), lacrosse: 9(3\%), rugby $8(2 \%)]$. The head/face accounted for the largest proportion of injuries (hockey: $25.4 \%$, football: $24.4 \%$, lacrosse: $33.3 \%$, rugby $50.0 \%$ ). Concussion was the most common injury in rugby $(50.0 \%)$ and football (24.4\%) and fractures the most common in hockey $(27.0 \%)$ and lacrosse (44.4\%). Contact with another player was the most frequently reported mechanism of injury (rugby: 87.5\%, football: $77.1 \%$, lacrosse: $66.7 \%$, hockey: 57.4\%), with most injuries related to contact by a player who was bigger or the same size as the injured player (hockey/rugby:100\%, lacrosse: 83.3\%, football: 81.5\%).

Conclusions Sport-related injuries in male collision sports are common, with four sports accounting for 33\% of all reported injuries across male Canadian high school sports. Head/face injuries were the most common, with the majority of injuries occurring due to contact with another player. There is scope to consider primary prevention strategies such as contact training and rule changes to address the risk of injury in youth collision sport.

\section{ABSTRACT WITHDRAWN}

\section{HIGH LEVEL OF ORAL DISEASE AND SELF-REPORTED PERFORMANCE IMPACTS IN MALAYSIAN ELITE ATHLETES}

${ }^{1}$ Norul Husna Mohammad-Hassan, ${ }^{1}$ Nora Sakina Mohd Noor, ${ }^{1}$ Nor Shafina Mohamed Nazari, 'Zubaidah Zanul Abidin, ${ }^{1}$ Noor Hayati Azami, 2,3 lan Needleman. 'Department of Restorative Dentistry, Faculty of Dentistry, University of Malaya, Kuala Lumpur, Malaysia; ${ }^{2}$ Centre for Oral Health and Performance, University College London Eastman Dental Institute, London, UK; ${ }^{3}$ UK IOC Research Centre, University College London, London, UK

\subsection{6/bjsports-2021-IOC.414}

Background Oral health can affect performance, including athletes who always need to be in their best conditions during training and competitions. However, oral health awareness has never been a priority among Malaysian athletes.

Objective This study primarily aimed to assess the association between oral health status and self-reported impact on athletes' sports performance, using the Oral Health Screening Toolkit for Athletes developed by University College London, UK.

Design/Setting/Participants This is a cross-sectional study using convenience sampling. Data were collected among elite athletes registered under the National Sport Council (NSC), Malaysia. 